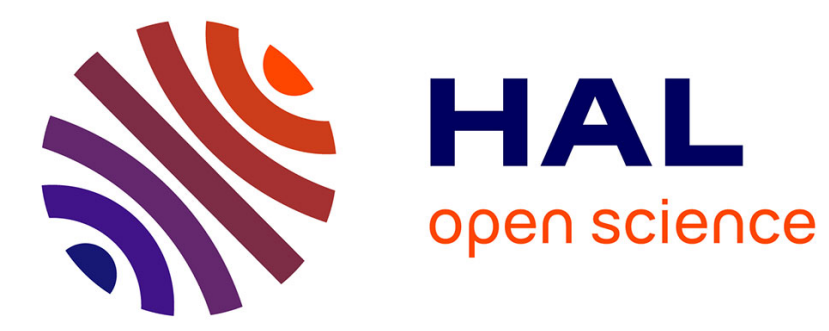

\title{
Experimental investigation of porosities evolution in a bonded assembly by means of X-ray tomography
}

\author{
Vincent Dumont, Claudiu Badulescu, J. Adrien, Nicolas Carrere, David
}

Thevenet, E. Maire

\section{To cite this version:}

Vincent Dumont, Claudiu Badulescu, J. Adrien, Nicolas Carrere, David Thevenet, et al.. Experimental investigation of porosities evolution in a bonded assembly by means of X-ray tomography. Journal of Adhesion, 2019, 10.1080/00218464.2019.1685984 . hal-02405487

\section{HAL Id: hal-02405487 \\ https://hal.science/hal-02405487}

Submitted on 3 Jan 2022

HAL is a multi-disciplinary open access archive for the deposit and dissemination of scientific research documents, whether they are published or not. The documents may come from teaching and research institutions in France or abroad, or from public or private research centers.
L'archive ouverte pluridisciplinaire HAL, est destinée au dépôt et à la diffusion de documents scientifiques de niveau recherche, publiés ou non, émanant des établissements d'enseignement et de recherche français ou étrangers, des laboratoires publics ou privés.

\section{(ㄷ)(1) $\$$}

Distributed under a Creative Commons Attribution - NonCommercial| 4.0 International 


\title{
Experimental Investigation of Porosities Evolution in a Bonded
}

\section{Assembly by means of X-ray Tomography}

\author{
V. Dumont ${ }^{1}$, C. Badulescu ${ }^{1}$, J. Adrien ${ }^{2}$, N. Carrere $^{3}$, D. Thévenet $^{1}$, and E. Maire ${ }^{2}$ \\ ${ }^{1}$ ENSTA Bretagne, UMR CNRS 6027, IRDL, F-29200 Brest, France \\ ${ }^{2}$ Univ. Lyon, INSA Lyon, CNRS UMR5 510 , Laboratoire MATEIS, F-69621, Villeurbanne Cedex, France \\ ${ }^{3}$ Safran Composites, 33 Avenue de la Gare, 91760 Itteville, France
}

September 9, 2019

Keywords: Porosity, Adhesively bonded assemblies, X-ray tomography, Coalescence

Corresponding author: vincent.dumont@ensta-bretagne.org

\begin{abstract}
Structral bonding is a very advantageous technique for lots of application fields such as aeronautics or marine industry, which require both advanced performances and lightweight structures. Nonetheless, adhesive joints are often subject to bonding defects: kissing bonds, uneven polymerization, or porosities within the material, for instance. These porosities, depending on their sizes and distributions, could jeopardize the mechanical strength of the assembly. Moreover, it is legitimate to hypothesize that these voids in the medium could be influenced by the application of a mechanical stress. In order to investigate this assertion, bonded samples are loaded by various tensile stress levels, and the porosities within the joint are visualized and characterized using in-situ X-ray microtomography. This paper deals with the evolutions of various quantities such as the number of porosities or their volumetric ratio along with the increasing load and with the diverse phenomena (nucleation, growth, coalescence, etc.) experienced during the testings. These results are extracted from the microtomographic data using a custom processing tool, whose parameters and performances are discussed.
\end{abstract}

\section{Introduction}

Structural bonded joints 1] represent today a promising technique due its main advantages such as: obtaining lightweight and complex structures, its simplicity of implementation, the possibility of unification of elements of different nature (in particular composite and metal), and providing a more homogenous distribution of the loading state in the two bonded elements. In recent years, a large number of studies 26 were carried out in the bonded joints field, both in terms of experimental characterization and numerical modeling of the mechanical behaviour of adhesive. 
Experimental characterization of adhesive requires the use of different types of tests dedicated to this area. Among the most common, one may include: (i) single lap joint test (SLJ), (ii) standardized thick adherend shear test (TAST) or (iii) modified Arcan test [5, 7].

SLJ is characterized by the simplicity of its samples and of the test, which only requires a simple test machine. However, this test is accompanied by significant stress concentrations at the ends of the overlap length, which may cause a significant scattering of the experimental results.

An alternative to this problem is the modified Arcan test, which diminishes drastically the stress concentrations close to the free edges of the adhesive. Also this test can generate different loading states in the joint such as: pure tensile, tensile-shear, pure shear or compressive-shear. However putting into practice the modified Arcan test involves a certain degree of difficulty, which makes it less usable in industrial environments. An alternative for the modified Arcan test is the modified Scarf test [8]. This test setup allows to generate a tensile or tension shear loads on an adhesive joint. The sample tested in this case is simple and can be used on standard tensile test machines without any additional devices. Moreover, this test is perfectly adapted for the fatigue tests investigation in the industrial environment.

Full field measurement of the displacement or strain by optical non-contact measurement systems, are increasingly being used in order to obtain the kinematic information necessary to characterize the mechanical behaviour 9. In most cases, the information obtained using non-contact measurement systems are obtained on the surface of the sample. Notwithstanding, it is necessary to get information inside the sample for the understanding of failure mechanisms and of their evolutions during the test.

In the last decade, several studies have been conducted, which highlight the major advantages of observations using X-ray tomography (XRT) 10 12. This tool is very advantageous because it allows to access the details of the microstructure of a material without its destruction. An original methodology is proposed 13 to estimate 3D displacement fields from pairs of images from X-ray computed microtomography. Contrary to local approaches, a global approach is followed herein that evaluates continuous displacement fields. Fast tomography combined with local crack driving force analysis has been employed to analyse crack-tip stress/strain singularities in an aluminium alloy $[11$. The application of fast microtomography has made possible to observe real crack initiation and propagation behaviours without intermediate unloading. The anisotropy of fracture toughness in AA2139 (AlCuMg) alloy sheet has been investigated via synchrotron radiation computed tomography of arrested cracks in Kahn tear test pieces for different loading cases [14]. More recent work [15] in the field of bonded joints have investigated the quantitative penetration of three coldset wood adhesives under hydraulic pressure into different types of modified wood using fluorescence microscopy and the results were compared to these of a previous study without pressure on adjacent wood samples. The three-dimensional visualization of the penetration of the adhesive into heat-treated Scots pine was also examined by X-ray tomography. X-ray micro tomography is used in 16 to visualize the distribution of melamineureaformaldehyde adhesive in the wood composite, particle board, and examine changes in adhesive distribution on wood particles (flakes) before and after pressing. Also micro X-ray computed tomography (XCT) was used to analyze the 3D adhesive penetration behaviour of different woodadhesive bondlines [17.

However, there are only few papers that analyze quantitatively the information that can be achieved 
using X-ray tomography in the field of structural bonded assemblies. It should be noted that the damage mechanisms are linked mainly by the presence of defects such as: porosities, lack of glue, the presence of several phases in the adhesive structure, etc.

The present paper focuses on the quantitative analysis at the micrometric scale of the bonded joints (epoxy adhesive and aluminum alloy). The main objective is to obtain the 3D microstructure of the adhesive after polymerization process (i.e. to identify the phases in the glue and their distribution) and to monitor the evolution of these phases when the sample is subjected to a tensile loading. In the first section, the experimental procedure will be presented, followed by the development and the validation of the adequate processing tool. Finally, the results will be outlined and discussed in the last section.

\section{Experimental procedure}

\section{$2.1 \quad$ X-ray tomography setup}

Since its first applications in the medical field [18, X-ray tomography has been the subject many studies both from a theoretical and applied point of view. A brief description is therefore given here in order to introduce the concepts, which will be used further in the text. Various experimental set ups can be used to perform X-ray tomography, but the basic principles of the technique remain the same (see Fig 11. Tomography measurements are based on the variation of the linear X-ray attenuation coefficient, hereafter written $\mu$, through the volume of a material. For a perfectly isotropic material this $\mu$ coefficient is a constant with respect to the spatial coordinates $(x, y, z)$. However, for non-homogenous material, such as porous environments, $\mu$ is a function of these coordinates. It is possible to obtain the spatial distribution of this $\mu$ coefficient by sending a source X-rays beam through the bulk of the studied sample, from different angles, and by collecting the transmitted beam. Hence, the sample is mounted on a rotator included in the tomograph chamber, and for each angular step, an X-ray beam is emitted, attenuated by the internal structure of the sample, and gathered by a detector. The attenuation phenomenon is described by a Beer - Lambert law depending on $\mu(x, y, z)$ :

$$
\frac{I}{I_{0}}=\exp \left(\int_{x_{0}}^{x_{\max }} \mu(x, y, z) d x\right)
$$

where $I_{0}$ represents the source intensity (emitted), $I$ is the detected intensity (transmitted and collected by the CCD) and $x$ represents distance along the transmission path $\left[x_{0} ; x_{\max }\right]$.

A series of $N$ radiograms, or radios, is obtained through the complete acquisition process (Fig 11. A reconstruction algorithm is then used to build the $3 \mathrm{D}$ internal structure of the sample (i.e. the spatial distribution of $\mu(x, y, z))$ from the radiographs series.

Those algorithms may be based on two different approaches: the software may either solve a set of linear equations to compute $\mu(x, y, z)$ (algebraic approach) or use a backprojection of the detected intensity by a Fourier transformation (analytical approach). Although the analytical approach is faster to compute, it requires a full dataset with no missing views from the radiographs series. This method is referred to as filtered backprojection reconstruction. For more details about these techniques and about their theoretical backgrounds, see 10,19 . The results presented in this paper were obtained by 


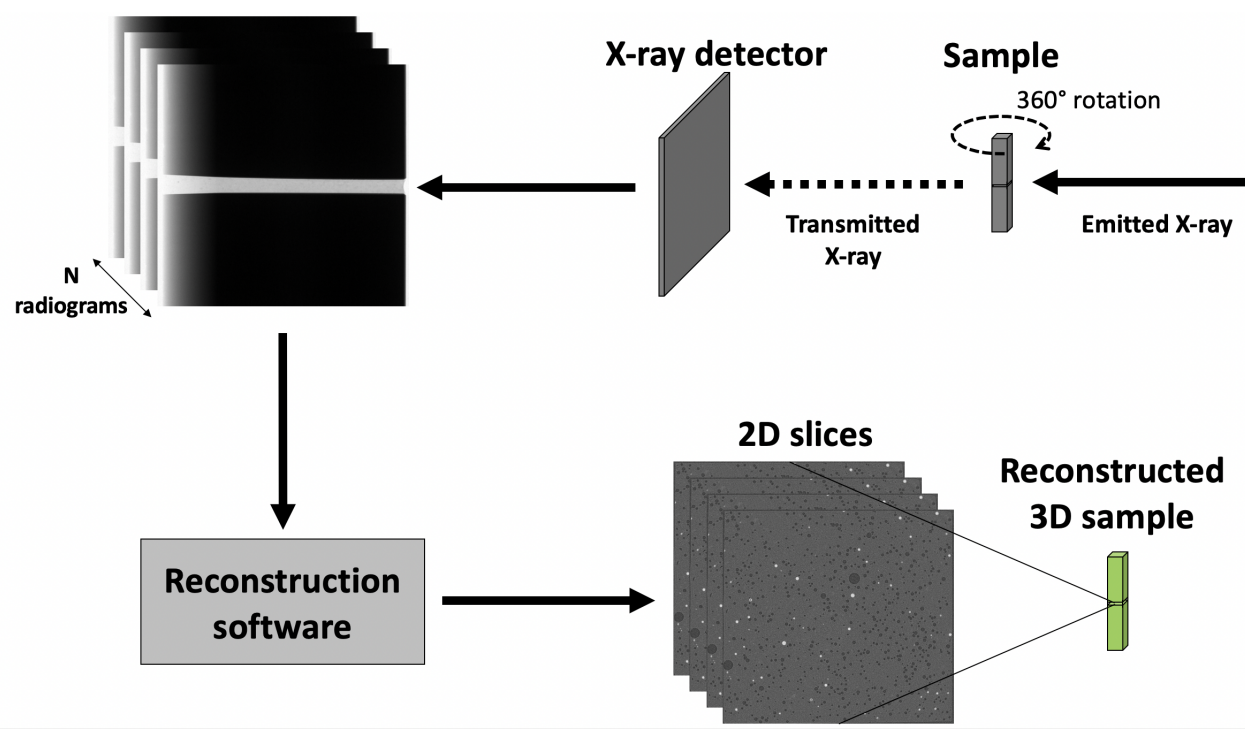

Figure 1: X-ray tomography principle

a filtered backprojection approach. Those algorithms depend on the X-ray source used to perform the measurements, being either a synchrotron or a laboratory tomograph, for the emitted beam itself depends on the underlying technology. For laboratory devices, the emitted beam is produced into a cone shape, rather than being constituted by parallel rays (which is the case for synchrotron X-ray sources). This has to be taken into account during the Fourier transformation backprojection [10. Although the formalism of the algorithm shall not be discussed here, the reader could find more information about this particular matter in [10, 19, 20]. The reconstruction step provides the 3D structure of the observed sample (Fig 1), defined with a given voxel size, in a similar fashion as pixels constitute a picture. It is worth noticing that the voxel size is related to the spatial resolution of the measurement, but is not equivalent. An empirical relationship between those quantities may be established, the spatial resolution being then twice the voxel size [10]. It is obvious that the spatial resolution of the experimental set-up depends not only on the voxel size, but also on various external parameters relative to the whole experimental set-up, and that the resolution must be in compliance with the characteristic sizes of the microstructure defects to observe. Some laboratory tomographs allow the operator to perform in situ measurements during mechanical and/or thermal solicitations 21. These kinds of experiments are though complex to handle due to the measurement artifacts it may produce. It is hence possible to access the microstructure of the sample, which is now a function of time, as opposed to classical, one-shot experiments delivering a unique radios series. The acquisition time for each radiographs series comes into play and defines the time resolution of the measurement, which has to be negligible when compared to the characteristic time of the observed phenomena. The following results in this paper are gathered using such an experimental set-up. 


\subsection{Sample preparation}

The samples consist in two Aluminum (2017A) substrates adhesively bonded by an epoxy resin layer of a given thickness. A simple, butt joint-like, geometry is designed as presented in Fig 2 The area of the bonded section is set to $6 \times 6=36 \mathrm{~mm}^{2}$. A non-uniform stress state is then expected within the bulk of the adhesive joint, along with stress concentration effects due to the influence of the edges of the substrates during the mechanical loading [1].

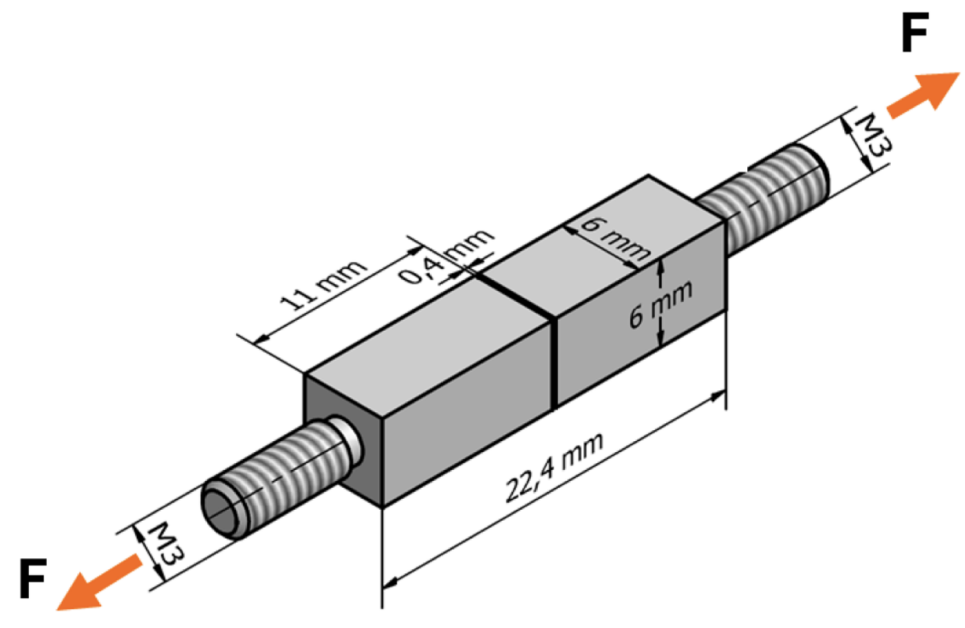

Figure 2: Sample geometry

The assemblies are obtained by bonding the substrates described above with the epoxy adhesive Huntsman ${ }^{T M}$ Araldite $420 \mathrm{~A} / \mathrm{B}$. The adhesive thickness is set to a value of $400 \mu \mathrm{m}$ and is controlled by a system of spacers. This system, and the specially designed corresponding apparatus, are presented in Fig2 2. This system enables both the control of the thickness of the adhesive joint and the correct alignment of the substrates. A clear space is also preserved in order to clean the adhesive joint once the substrates are assembled, and thus to obtain a neatly defined sample.

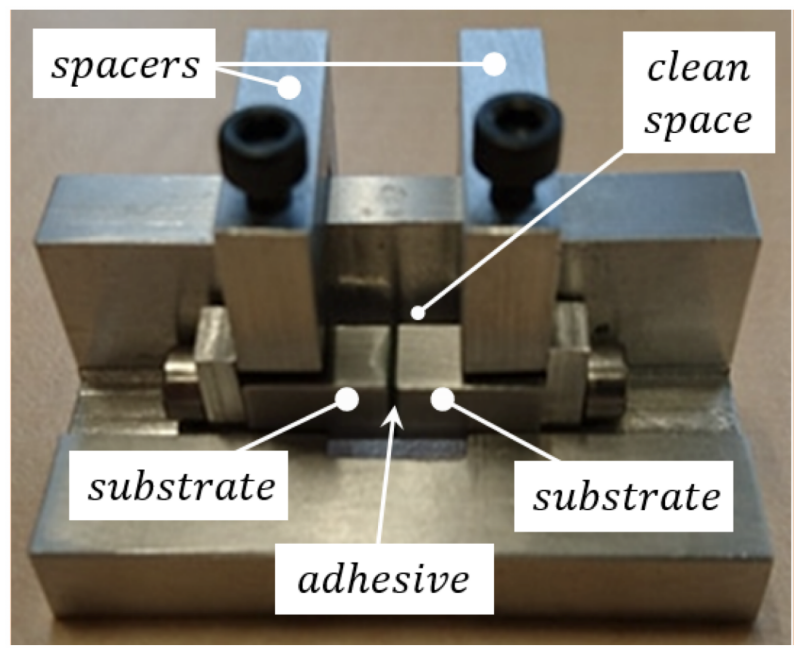

Figure 3: Sample mounted in the bonding apparatus with controlled joint thickness 
In order to guarantee the good adhesion between the adhesive layer and the substrates, a classical (chemical and mechanical) surface treatment is performed on the bonding surfaces to remove any greasy byproducts that may remain after the machining of the substrates and to create an adequate surface roughness for the desired mechanical interlocking between the substrates and the adhesive. In a first step, the substrates are placed in an acetone bath for several hours to degrease the surfaces. They are then treated with grade 180 sandpaper to remove the remaining impurities and to eliminate any oxide layer that could have formed due to the exposition of the Aluminum to the ambient environment. Moreover, this mechanical treatment creates the aforementioned surface roughness required for a good adhesion. Lastly, the surfaces thusly treated are cleaned with acetone to remove the impurities and Aluminum particles created with the mechanical treatment. The adhesive joint is then prepared by applying the mixed epoxy resin and hardener on both substrates, which are assembled and installed in the apparatus (Fig 3) carefully adjusted to obtain the desired joint thickness. The samples are finally placed in a Secasi Technologies ${ }^{T M} 100 / 60$ thermal enclosure at $115^{\circ} \mathrm{C}$ for 1 hour to ensure a fully polymerized material. Due to the very limited volume of the adhesive joints, it is assumed that the thermal field in the material is homogeneous, and that there is no significant influence of possible curing degree gradients in the adhesive. It can be seen in Fig, 2 that threaded holes are machined at each end of the samples to allow the positioning of the rods necessary to the installation of the samples in the clamping jaws of the tensile machine to be used. Those holes are also used during the gluing process to control the relative positioning of the substrates.

\subsection{Test procedure}

The samples are placed inside a Phoenix ${ }^{T M}$ VtomeX tomograph equipped with a Varian Paxscan ${ }^{T M} \mathrm{X}-$ Ray detector featuring a resolution of 1920x1536 pixels. This detector outputs a 14-bits coded grayscale picture of the attenuation. The chamber of the tomograph includes a $3 \mathrm{kN}$ tensile machine, which allows to perform X-ray tomography measurements while applying a mechanical load to the sample (Fig 4). The voxel size obtained using this experimental set-up is $6 \mu \mathrm{m}$ x $6 \mu \mathrm{m} \times 6 \mu \mathrm{m}$. The corresponding spatial

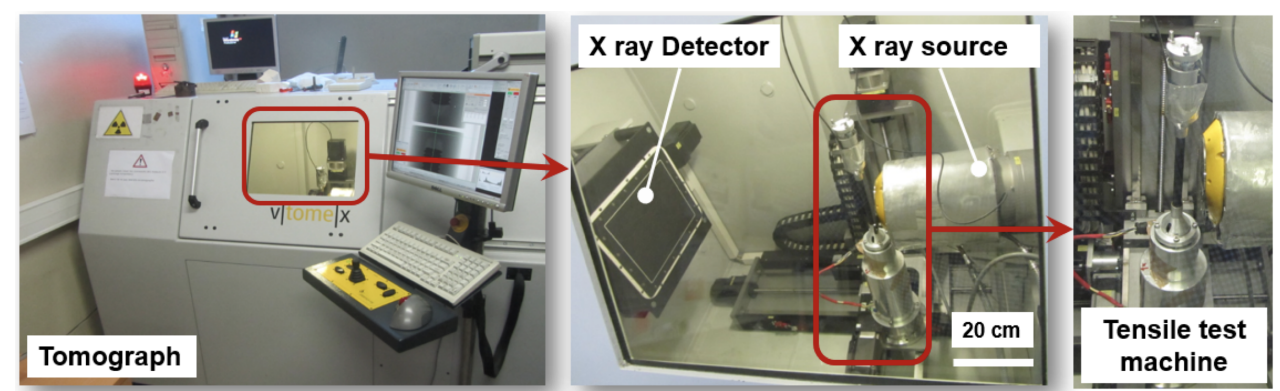

Figure 4: Standard laboratory Phoenix ${ }^{T M}$ VtomeX tomograph

resolution is expected to be in compliance with the characteristic size of the porosities to be observed. Also, the adhesive contains $0.16 \%$ (weight ratio, according to manufacturer data) of glass beads, whose diameters are about $80 \mu \mathrm{m}$, i.e. more than 10 times greater that the resolution of the device. The mea- 
surements are assumed to occur for a quasi-static state of the samples, and therefore the corresponding characteristic time overshadows the acquisition time for a radiographs series. Hence, it is concluded that the experimental set-up is well-suited to the measurements to be performed. In order to reconstruct the full observed volume, the acquisition is performed along with a rotational motion of the sample. A total of 912 pictures are taken throughout the rotation, for a complete acquisition time of 12 min. These series are then used as previously mentioned by an algorithm based on the results presented in [4], in the perimeter of an analytical approach of the computation of the spatial field $\mu(x, y, z)$. The full volume of the samples, visualized by means of the variation of the values of the attenuation coefficient $\mu(x, y, z)$, is then broken into a series of slices (Fig, 6).

An amount of 96 slices is obtained for the adhesive joint only, from the tomographs series. As already mentioned, the tensile test machine included in the tomograph chamber allows us to perform these measurements while applying a mechanical loading to the samples. The decision has been made to incrementally increase the applied load with a $200 \mathrm{~N}$ step (Fig.8). The ramps between the steps are displacement-controlled $(0.5 \mathrm{~mm} / \mathrm{min})$. A stress-strain curve for the considered material is given in Fig $5 \mathrm{~b}$ along with the stress states corresponding to each aquisition step (Fig 5a. In addition, the elastic properties of the adhesive are presented in Table 1.

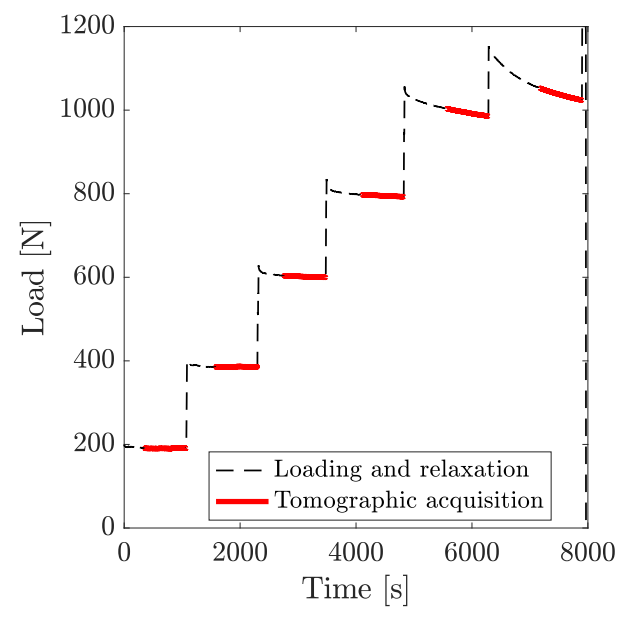

(a) Load applied to the sample

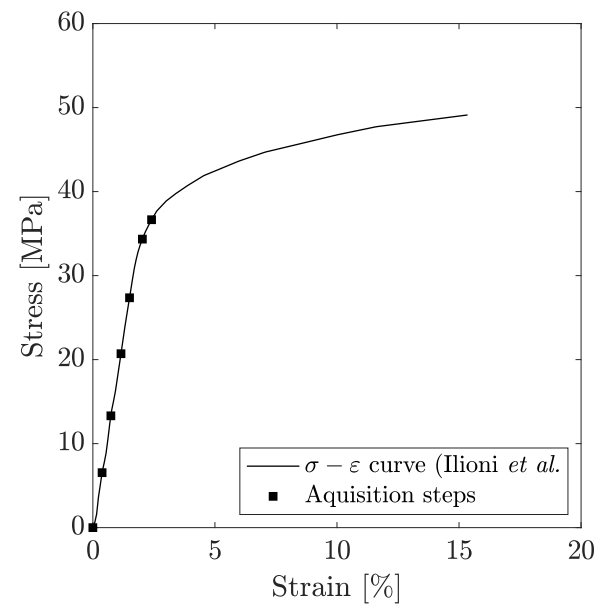

(b) Tensile stress-strain curve of the considered adhesive 22

Figure 5: Mechanical loading and properties of the adhesive

\begin{tabular}{ll}
\hline Parameter & Value \\
\hline$E[\mathrm{MPa}]$ & $2000 \pm 50$ \\
$\nu[-]$ & $0.41 \pm 0.01$ \\
\hline
\end{tabular}

Table 1: Elastic parameters of the adhesive 22

The process goes on until the failure of the sample. It is clear from Fig 5 a that stress relaxation phe- 
nomena are occurring during these steps, especially for the high loads. For each step, the corresponding displacement is therefore maintained for a certain time, in order to let these phenomena occur. Otherwise, the measurement would be disturbed by the on-going relaxation, due to the similarity between the acquisition time and the relaxation characteristic time. By considering significantly longer load steps, it is possible for the load to reach an asymptotical value and hence to obtain a relatively steady state during the 12 minutes necessary to complete a full rotation of the sample. The aforementioned asymptotical value is harder to reach in a reasonable time the higher the load is, and may even not be reachable in some cases (loads superior to $800 \mathrm{~N}$, Fig $5 \mathrm{a}$. This may lead to blurry acquisition data, as those measures are critically sensitive to any disturbances, which cause significant artifacts. It is then possible to acquire a snapshot of the 3D microstructure of the adhesive joint for several loads. In other words, given the adequate post-processing tools, it is possible to follow the evolution, along with the applied load, of the microstructure, in a non-destructive in-situ fashion.

\section{Postprocessing tool}

A time series of radiographs sets is delivered by the experiments detailed above. The reconstructed volume of the central part of the sample for the initial state of load is showed in the Fig.7a. Those raw grayscale images, display 3 visible phases (see Fig 6b to Fig 6d): the adhesive, the air porosities and the glass beads introduced by the manufacturer.

The raw data is initially encoded in 16 bits. In order to reduce the volume of data and thus facilitate post-processing steps, the raw 16-bits data were converted to 8-bits grayscale levels. This step results in a lighter dataset and in faster processing steps without damaging excessively the quality of the data to observe. The data obtained with X-ray tomography were then post-processed in order to extract the following information: the spatial distribution and rate of each phase as well as their evolution during the different load applied. The complete study of the microstructure with respect to the applied load requires the development of a processing tool, which will: (i) eliminate unwanted artifacts from the reconstructed slices of adhesive and extract the mean intensity;(ii) filter the signal to remove the noise and (iii) detect the microstructural entities observed (here, porosities and glass beads) from a grayscale intensity threshold.

This processing tool should allow to automatically detect the requested microstructural entities from the raw signal presented in Fig $7 \mathrm{~b}$.

\subsection{Creation of synthetic microtomographic data}

In order to design the processing tool, a preliminary study is performed using synthetic microtomographic data. The main advantage of this technique is that every property of the microstructure is accurately known, since it is imposed. The grayscale levels of the voxels for a $2 \mathrm{D}$ slice taken in this synthetic volume are given in Eq.2.

$$
S_{z_{j}}(x, y)=I_{0, Z_{j}}(x, y)+\gamma_{i} \cdot p_{i}(x, y)+b_{z_{j}}(x, y)
$$




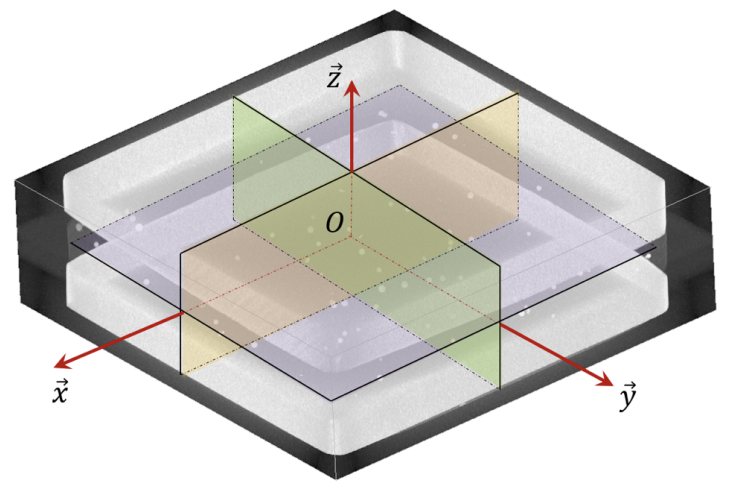

(a) Central part of the sample reconstructed

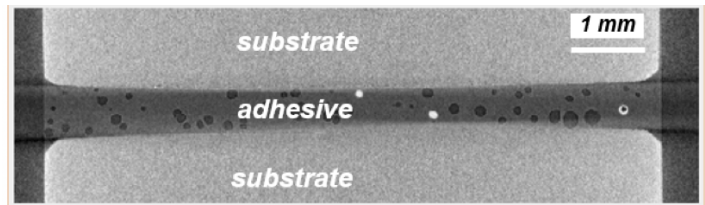

(c) $\mathrm{xOz}$ slice in the middle of the adhesive

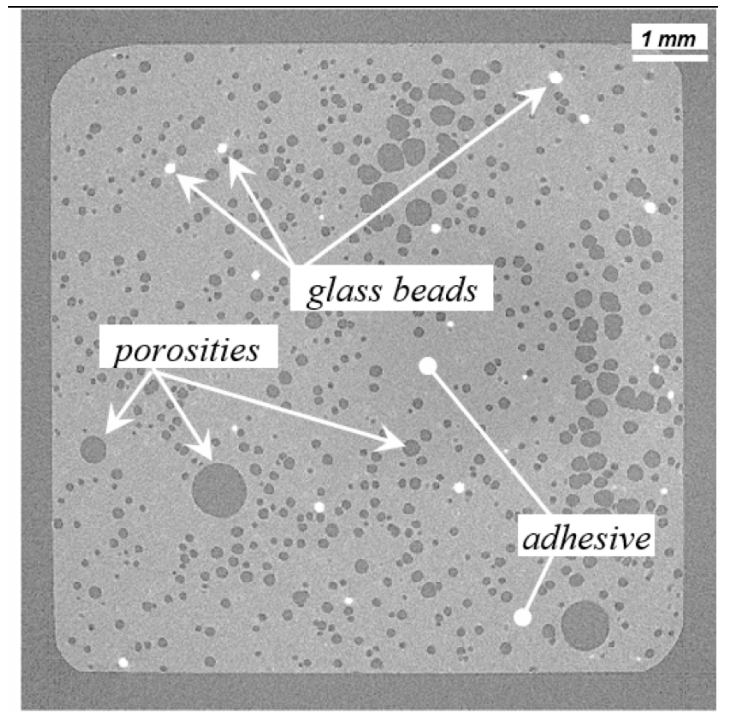

(b) $\mathrm{xOy}$ slice in the middle of the adhesive

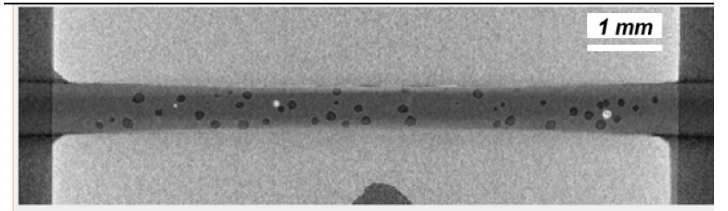

(d) $\mathrm{yOz}$ slice in the middle of the adhesive

Figure 6: Reconstructed volume of the sample obtained from tomography investigation

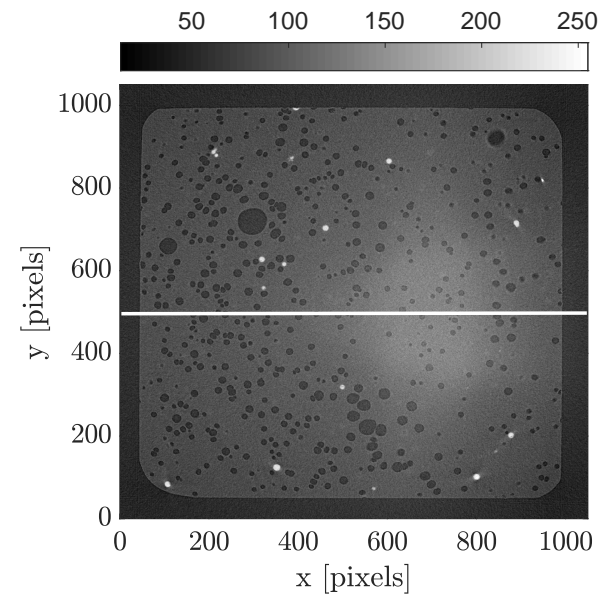

(a) Unprocessed volume slice close to the substrate adhesive

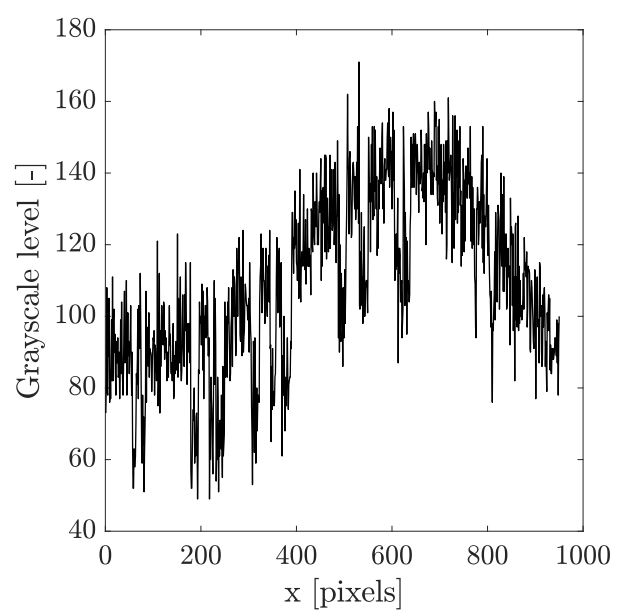

(b) Grayscale values along a path (white line in Fig 7 a

Figure 7: Slice of raw data

where $S_{z_{j}}(x, y)$ is the grayscale levels of the slice $j$ in the $Z$ direction with $j \in[1,65], I_{0, Z_{j}}(x, y)$ is the mean grayscale level, $\gamma_{i}$ is the contrast for each phase $i, p_{i}(x, y)=N_{i}$ with $N_{i} \in\left[0,2^{8}-1\right]$ are 8-bits grayscale levels for each phase $i$, and $b_{z_{j}}(x, y)$ is a 8-bits grayscale level correspondong to Gaussian measurement noise. 
The intensity of the measurement noise is assumed to be independant of the position. Therefore, the standard deviation of the Gaussian distribution used to generate the measurement noise is the same in the entire volume. The value of the standard deviation $\sigma$ is experimentally obtained, by subtracting two microtomographic acquisitions of the same volume. The properties of the generated volume are displayed in Table 2 .

\begin{tabular}{ll}
\hline Property & Value \\
\hline Size & $960 x 960 x 65$ voxels \\
Porosities number $n_{p_{t h}}$ & 3114 (non-overlapping) \\
Porosities ratio $p_{t h}$ & $9.69 \%$ \\
Porosities diameters & Uniformly distributed between 1 and 35 voxels \\
Noise intensity & Standard deviation $\sigma=9.62$ \\
\hline
\end{tabular}

Table 2: Synthetic data properties

The spatial distribution of the generated porosities can be visualized in Fig 8a. Moreover, the distribution of their corresponding diameters is available in Fig. $8 \mathrm{~b}$. One may notice that the diameters distribution is not perfectly uniform. This is due to the non-overlap condition for the porosities used during the generation of the volume. Therefore, a few of the randomly generated diameters (mainly the largest ones) were programmatically modified or discarded in order to comply with this constraint.

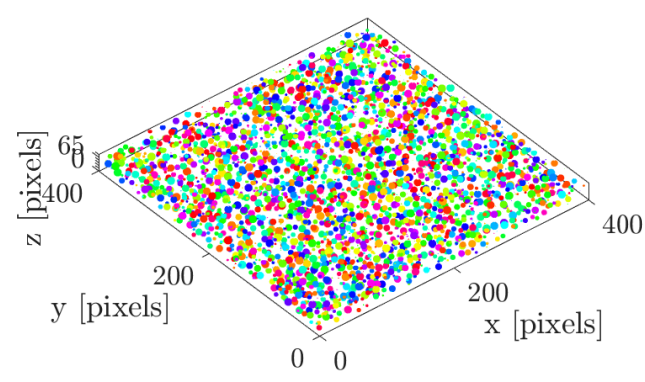

(a) Spatial distribution of porosities

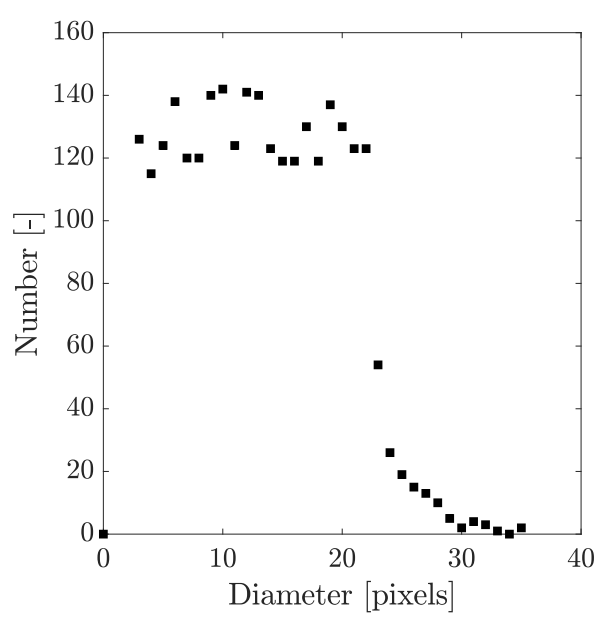

(b) Equivalent diameters distribution in the synthetic volume

Figure 8: Artificially generated volume for known porosities amount, porosity ratio and porosities locations 


\subsection{Postprocessing tool architecture}

In order to characterize the porosities located in a volume, it is necessary to segment the tomographic data. To do so, a processing tool is designed and programmed using Matlab ${ }^{\mathrm{TM}}$ software. The scheme of treatment of the raw data is showed in Fig 9 .

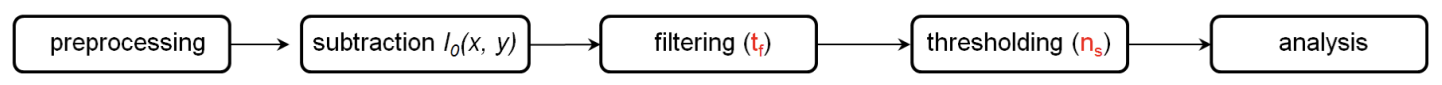

Figure 9: Processing tool architecture

\subsubsection{Preprocessing}

As it may be seen in Figs $6 \mathrm{~b}$ to $6 \mathrm{~d}$, the radiograms include a large part of the substrates and some blank space around the samples. Hence, the reconstructed volume also contains these elements, resulting in some useless volume slices (with only Aluminum showing). The adhesive being absent from these pictures, they are removed from the dataset. By doing so, it is possible to reduce the number of slices to be analyzed from several hundreds to 65 .

\subsubsection{Subtraction of $I_{0}$}

The mean grayscale intensity is assumed to follow a polynomial expression, as in Eq3. The degree of this polynomial surface is left undetermined in Eq 3 . In pratice, $m=3$ was enough for the study presented in this paper. The value of $m$ may be adjusted accordingly with the shape of $I_{0}$.

$$
I_{0}(x, y)=\sum_{j=0}^{m} \sum_{i=0}^{j} c_{i, j-i} x^{i} y^{i-j}
$$

The coefficients $c_{i, j-i}$ in Eq 3 are identified using the grayscale levels of each reconstructed volume slice (Fig 10a . The obtained values of $I_{0}$ are then subtracted to the grayscale levels of the volume (Fig 10b), in order to obtain a flattened signal (Figs $10 \mathrm{c}$ and $10 \mathrm{~d}$ ).

\subsubsection{Filtering and thresholding}

The filtering and the thresholding steps are designed to smooth the signal (i.e. remove as much noise as possible from the flattened signal) and to identify precisely the transition between the adhesive and the porosities in terms of grayscale levels (Fig, 11). To correctly tune the corresponding parameters $\left(t_{f}\right.$ and $n_{s}$, see Fig 9 , an optimization loop is performed using $M a t l a b^{T M}$. The filter used in the process is a classical 2D median filter 23, characterized by its size $t_{f}$. The main advantage of this formulation is that it is edge-preserving 24] (i.e. it will preserve the edges between the adhesive and the porosities), which is paramount to accurately detect the porosities included in the adhesive.

The threshold $n_{s}$ is trivially defined as the minimal grayscale level shift to consider to detect a microstructural phase switchover. Those values are then adjusted in order to minimize an error function, characterizing the performance of the tool. It is possible to consider two different shapes for this error 


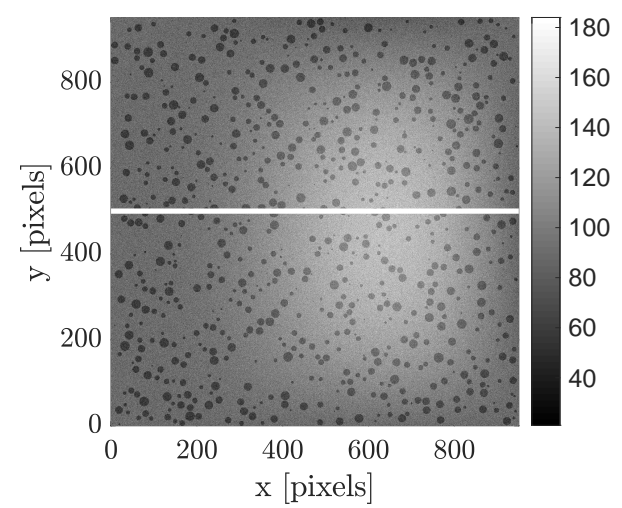

(a) Artificially generated adhesive slice before subtraction of $I_{0}$

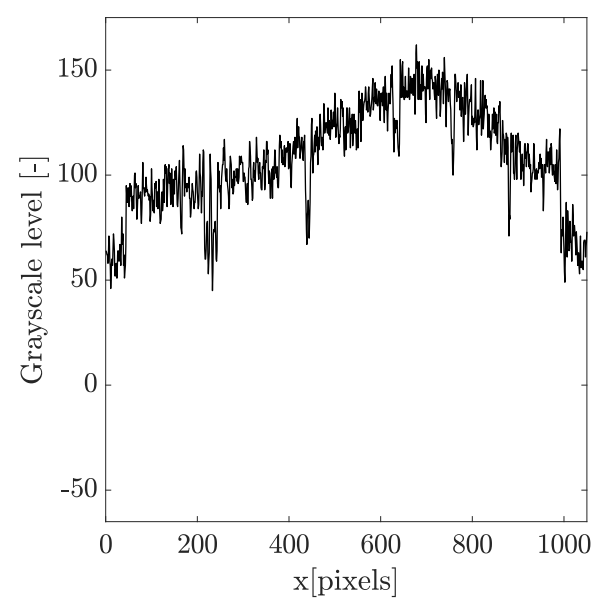

(c) Grayscale levels along $y=500 \mathrm{px}$ for the raw signal (white line in Fig 10a

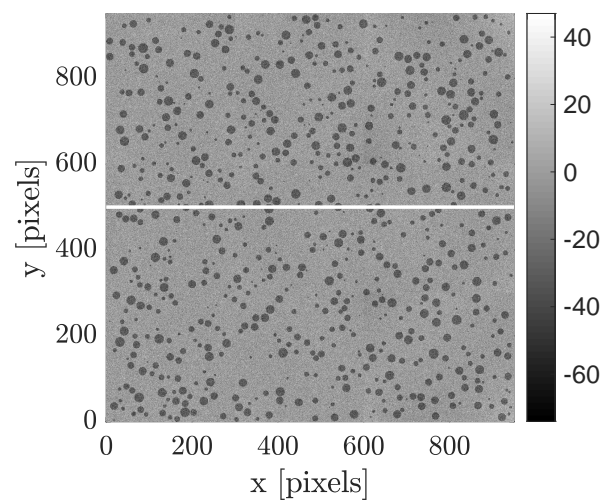

(b) Artificially generated adhesive slice after subtraction of $I_{0}$

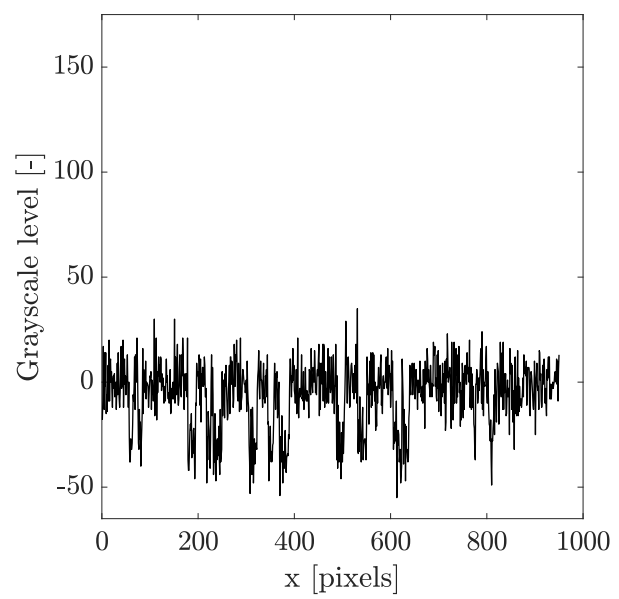

(d) Grayscale levels along $y=500 \mathrm{px}$ for the flattened signal (white line in Fig $10 \mathrm{~b}$ )

Figure 10: Subtraction of $I_{0}$

function: $\operatorname{Err}_{M 1}$ which takes into account only the difference between the porosity ratios $p_{i}$ (method M1, Eq 4 and $\operatorname{Err}_{M 2}$ which takes into account the difference between the porosity ratios $p_{i}$ and the difference between the number of porosities detected $n_{p_{i}}(\operatorname{method} \mathrm{M} 2, \mathrm{Eq} 5$ ).

$$
\begin{gathered}
\operatorname{Err}_{M 1}\left(t_{f}, n_{s}\right)=\left[\frac{p_{t h}-p_{o b t}\left(t_{f}, n_{s}\right)}{p_{t h}}\right]^{2} \\
\operatorname{Err}_{M 2}\left(t_{f}, n_{s}\right)=\alpha_{1}\left[\frac{p_{t h}-p_{o b t}\left(t_{f}, n_{s}\right)}{p_{t h}}\right]^{2}+\alpha_{2}\left[\frac{n_{p_{t h}}-n_{p_{o p t}}\left(t_{f}, n_{s}\right)}{n_{p_{t h}}}\right]^{2}
\end{gathered}
$$

where $p_{t h}$ and $p_{o b t}$ are respectively the imposed porosity ratio and the detected porosity ratio for a given $\left(t_{f}, n_{s}\right)$ couple, and $n_{p_{t h}}$ and $n_{p_{o b t}}$ are respectively the imposed porosities number and the detected porosities number for a given $\left(t_{f}, n_{s}\right)$ couple.

The method M1 defines the error between the generated porosity field and the detected porosity field as a function of the porosity ratios whereas the method M2 defines a more extensive error function as a 


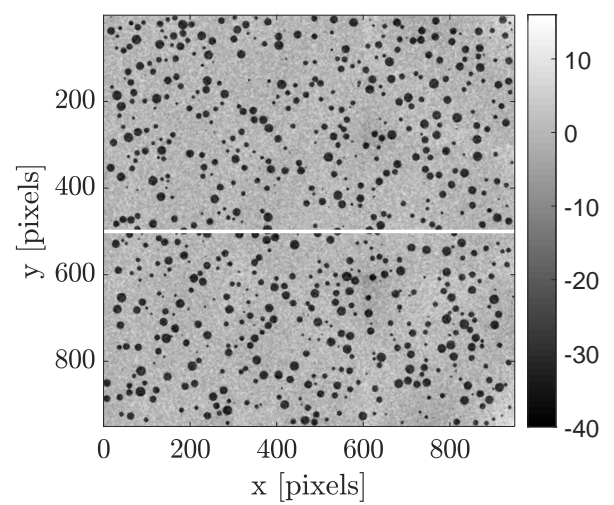

(a) Artificially generated slice after filtering

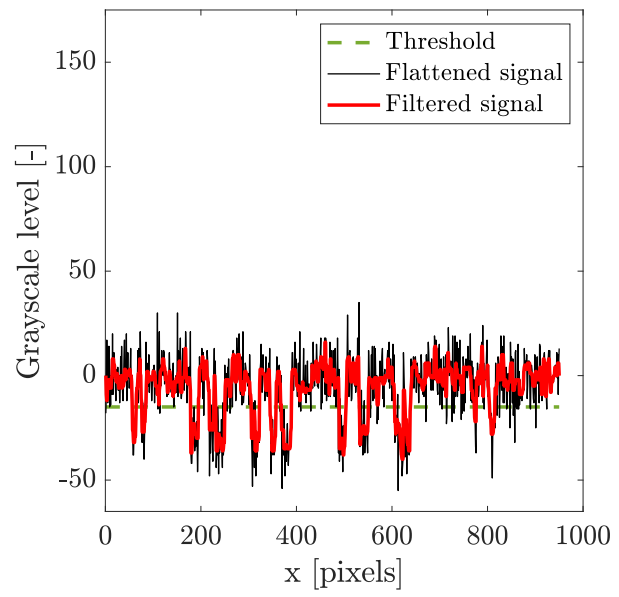

(b) Artificial grayscale levels along $y=500 \mathrm{px}$ for the flattened signal

Figure 11: Filtering of the synthetic data

function of both the porosity ratios and the porosities amounts (Eq4 and Eq 5 . In the M2 formulation, a couple $\left(\alpha_{1}, \alpha_{2}\right)$ is introduced so it is possible to assign different weights to each one of the two terms if need be. By default, these values are set to 0.5, so as to obtain an equivalent weight for each term in Eq.5. The optimization loop is performed in a first stage for the M1 approach, and delivers a first set $\left(t_{f}, n_{s}\right)_{M 1}$ identified as the parameters corresponding to the lowest error value $\left(t_{f_{M 1}}=3\right.$ and $\left.n_{s_{M 1}}=-15\right)$ easily pinpointed on the error map (Fig 12a). The loop is then repeated for the M2 approach, which delivers two error maps (Figs. $12 \mathrm{~b}$ and $12 \mathrm{c}$ ) instead of one, as two terms are used to compute the error. By combining the minimum valleys obtained through the M2 method, it is possible to compute the global error for this approach (Fig 12d). Due to the removal of $I_{0}$ from the baseline signal (Figs 10c and fig:rmI04), a negative value is obtained for $n_{s}$. It is therefore not a 8-bits grayscale level anymore, but a grayscale intensity. 


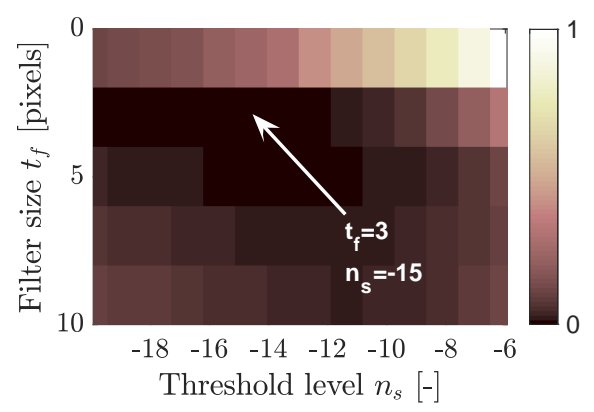

(a) Error map obtained for the M1 method as a function of $t_{f}$ and $n_{s}$

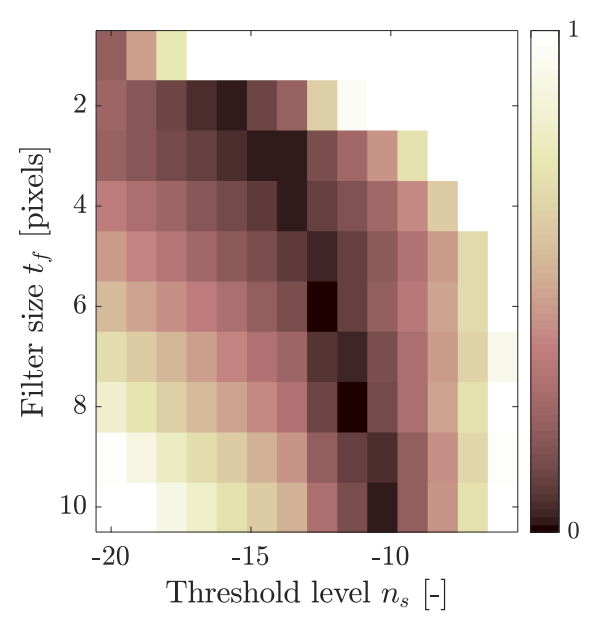

(c) Error map relative to the porosity ratio for the M2 method as a function of $t_{f}$ and $n_{s}$

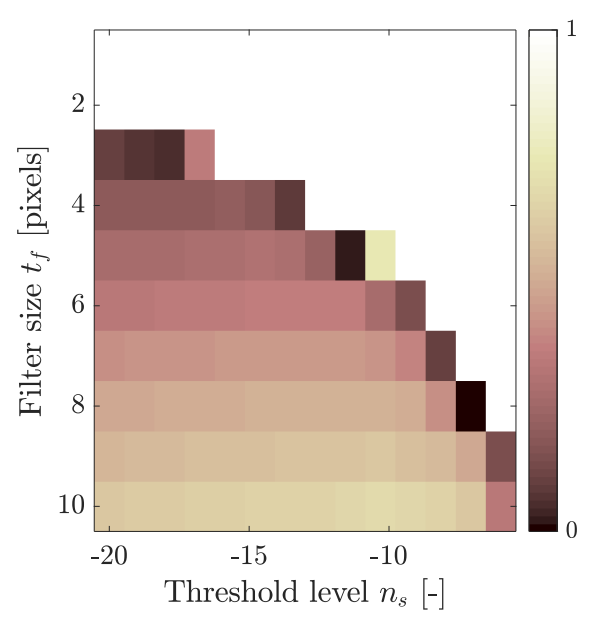

(b) Error map relative to the number of porosities for the M2 method as a function of $t_{f}$ and $n_{s}$

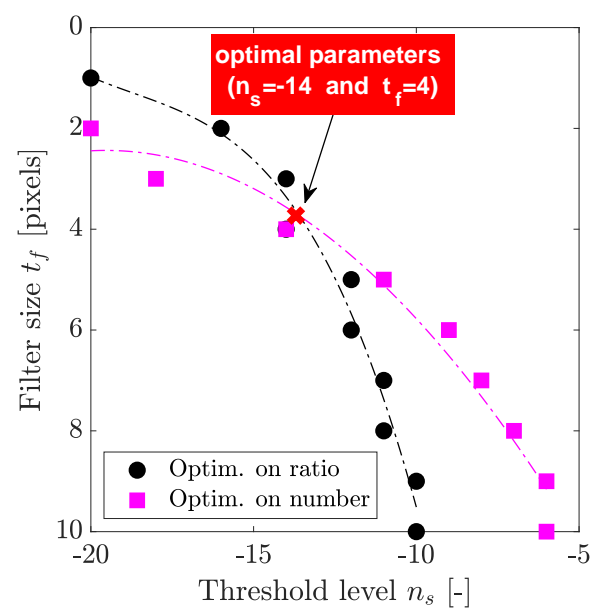

(d) Optimal parameters for the M2 method

Figure 12: Identification of the $\left(n_{s}, t_{f}\right)_{M 2}$ set

From the intersection of these domains (Fig 12d), it is possible to extract a second parameters set $\left(t_{f}, n_{s}\right)_{M 2}$, which outputs not only the correct porosities volume fraction, but also the correct porosities amount. From these observations, it can be concluded that the processing tool designed in this paper allows for a correct evaluation of the porosity distribution in an adhesive layer, in terms of both spatial distribution and radius distribution. 


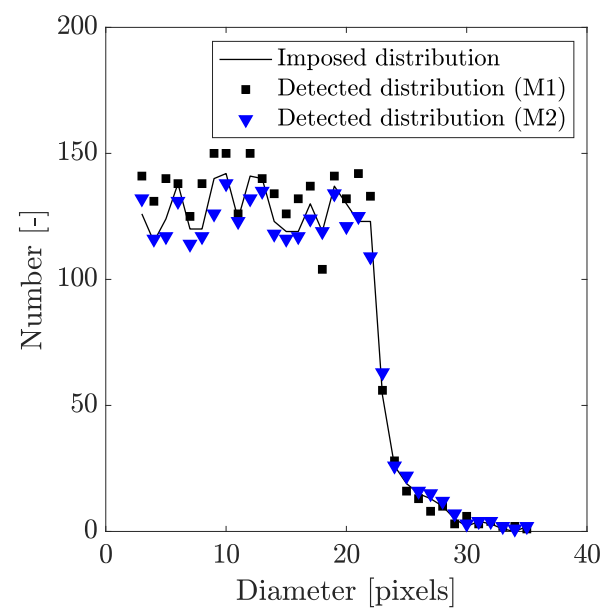

Figure 13: Comparison between methods M1 and M2 in terms of porosity detection

It is clear from Fig 13 that the M1 method is inefficient to accurately detect the correct number of porosities, especially for the lowest diameters. This inefficiency tends to fade along with the increase of the equivalent diameters of the porosities to be detected. The M2 method displays results of overall higher quality, and is less prone to be disturbed by very small porosities. This method allows the user to accurately detect the voids in the adhesive, with a fairly high accuracy in terms of both number and equivalent diameter.

The tool, successfully calibrated here, was used on a generic adhesive volume including a porosities field whose diameters are randomly distributed in a given range representative of the actual configuration. To be perfectly in compliance with a real experimental case, these diameters should be normally distributed. However, this slight inadequacy is expected to be of negligible influence, as it seems from Fig 13 that the designed tool outputs the correct number of porosities for nearly every equivalent diameter. The only difference between a normal and a random distribution being the number of entities for each equivalent diameter, one should expect that the processing tool would correctly estimate the voids field for a different distribution.

\subsection{Influence of measurement noise on porosities detection}

Although it appears that the two approaches presented give similar results, they can be evaluated in a more extensive fashion by studying the influence of the picture noise level on the porosity field detected. To perform this comparison, the two resulting tools are applied to five cases with five noise draws (Fig 14 ). From Fig $14 \mathrm{a}$ it is obvious that even though the two methods presented previously give similar results in terms of porosities volume fractions (which is slightly underestimated due to the voxelization of a continuous media), only the M2 approach is able to deliver a correct estimation of the porosities amount (Fig 14b in a given volume. It is then concluded that the M2 method should be used, for $t_{f}=4$ and $n_{s}=-14$. 


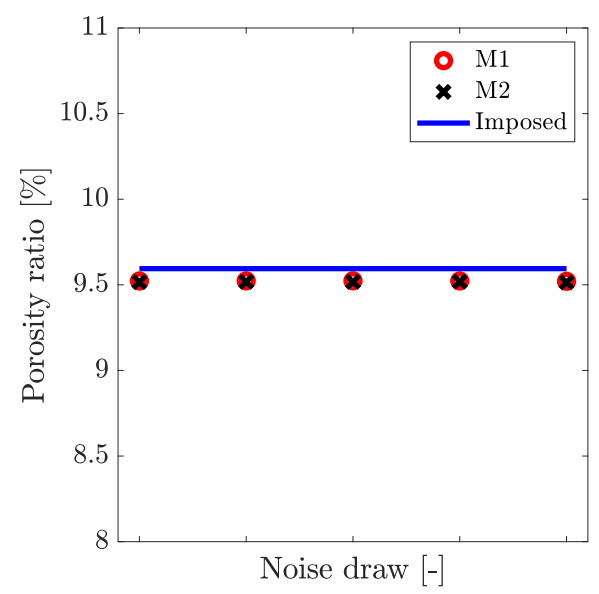

(a) Effect of the noise on the porosity ratio

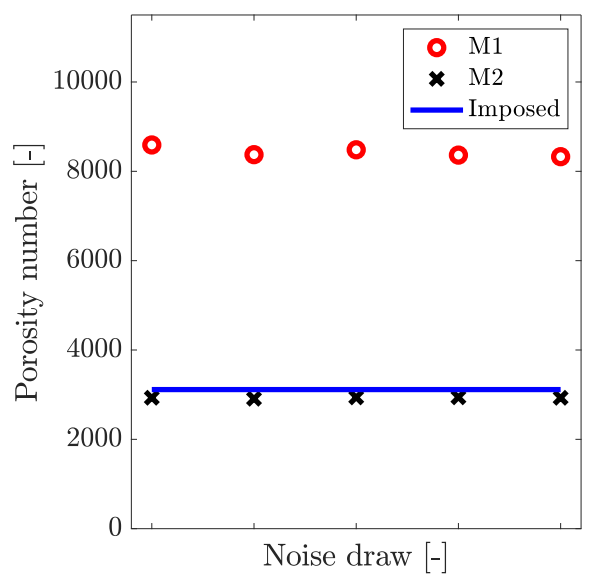

(b) Effect of the noise on the number of porosities

Figure 14: Comparison between the results delivered by the M1 and M2 approaches for 5 different noise draws

The obtained results are summarized in Tables 3 and 4 , where $\bar{i}$ and $\sigma_{i}$ are resctively the mean value and the standard deviation of the quantity $i$.

\begin{tabular}{lccc}
\hline & $p_{t h}[\%]$ & $\overline{p_{o b t}}[\%]$ & $\sigma_{p_{o b t}}[\%]$ \\
\hline Theoretical & 9.59 & - & - \\
M1 & - & 9.522 & $1.05 .10^{-3}$ \\
M2 & - & 9.516 & $1.80 .10^{-3}$ \\
\hline
\end{tabular}

Table 3: M1 and M2 methods results compared to the theoretical porosity ratio

\begin{tabular}{lccc}
\hline & $n_{p_{t h}[-]}$ & $\overline{n_{p_{o b t}}[-]}$ & $\sigma_{n_{p_{o b t}}[-]}$ \\
\hline Theoretical & 3114 & - & - \\
M1 & - & 8427.2 & 107.7 \\
M2 & - & 2930.1 & 12.7 \\
\hline
\end{tabular}

Table 4: M1 and M2 methods results compared to the theoretical number of porosities

Even though fairly similar results are obtained in terms of porosity ratio $p_{o b t}$ with both methods (Table 3), it is clear from Table 4 that the M1 method is not able to accurately detect the correct number of porosities in the artificial datasets. Quantitatively, the error on the dectected number of porosities is above $170 \%$ using M1, whereas it is only of $6 \%$ using M2. This is explained by the fact that M1 tends to detect erroneous porosities for small diameters (Fig 13 ). 


\section{Results and discussion}

\subsection{Evolution of the porosities number and of the porosity ratio with respect to the applied load}

From the observations made regarding the two methods presented above, it was chosen to use the M2 methodology, with an optimized $\left(t_{f}, n_{s}\right)_{M 2}$ couple equal to $(4,-12)$.

An immediate output of the processing tool presented in the previous section is the porosities-to-adhesive volume ratio. It is then possible to track the evolution of this quantity along with the increase in the applied load (Fig 15a).

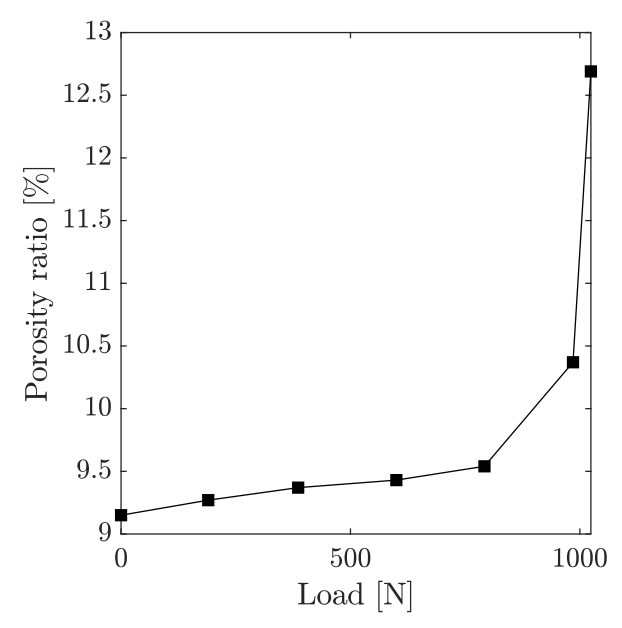

(a) Evolution of the porosity volume fraction with respect to the applied load

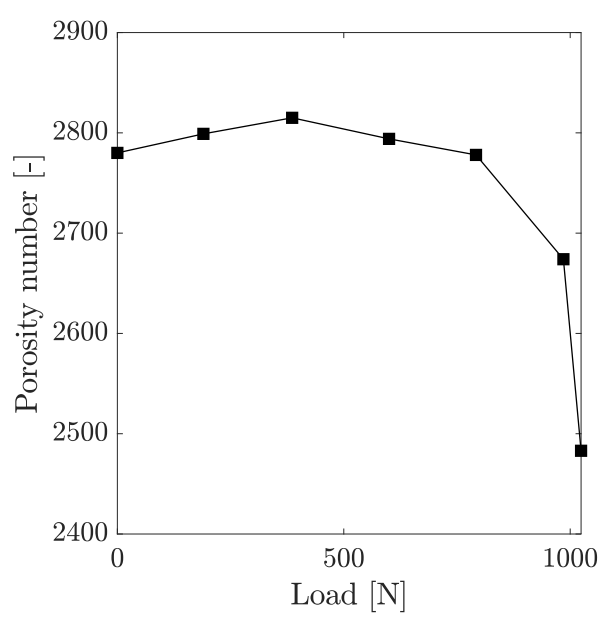

(b) Evolution of the number of detected porosities with respect to the applied load

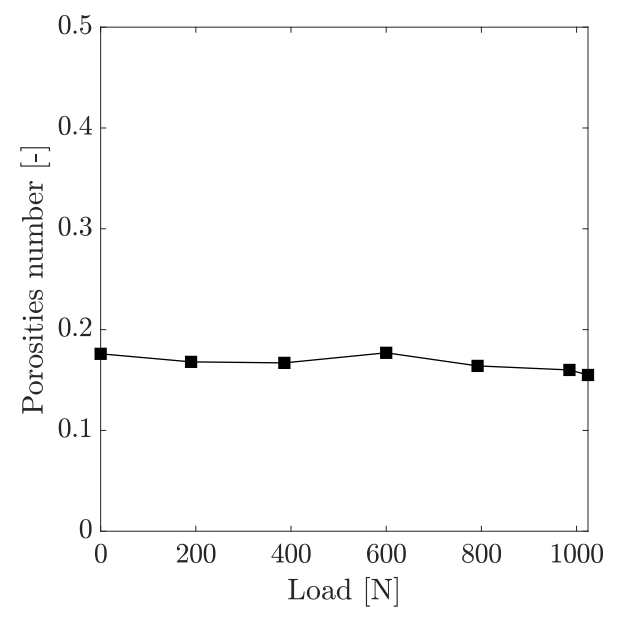

(c) Glass beads ratio with respect to the applied load

Figure 15: Changes undergone by the internal microstructure of the adhesive joint along with the increasing load

In addition, by means of the M2 method defined by the Eq 5 it is possible to obtain a fairly accurate 
estimation of the number of porosities included in the adhesive layer, which may be plotted versus the increasing load as well (Fig 15b).

It appears from these data that the mechanical solicitation applied to the sample may lead to significant changes in the microstructural properties of the adhesive. Both these quantities seem to follow a trend defined by two distinct domains: $F \leqslant 800 \mathrm{~N}$, for which the evolution seems fairly linear with respect to the applied load; and $F \geqslant 800 \mathrm{~N}$, for which the evolution becomes non-linear as a function of the applied load, with a sudden increase in porosity volume fraction, and with a sudden decrease in porosities number.

It is possible to validate the results presented above by verifying that the developed method outputs the correct glass beads ratio, which is given by the manufacturer.

It appears from Fig $15 \mathrm{c}$ that this quantity remains almost constant, as it should be since there is obviously no external glass beads input. It is possible to compute the mean $\overline{p_{\text {beads }}}$ of these values, to be compared to the manufacturer data:

$$
\overline{p_{\text {beads }}}=0.167 \%
$$

These numerical values are fairly consistent with the numbers provided by the manufacturer regarding this adhesive, since the adhesive theoretically contains $0.16 \%$ of beads (in terms of volume).

Finally, the distribution of the diameters of the detected glass beads may be computed. This distribution is shown in Fig, 16 .

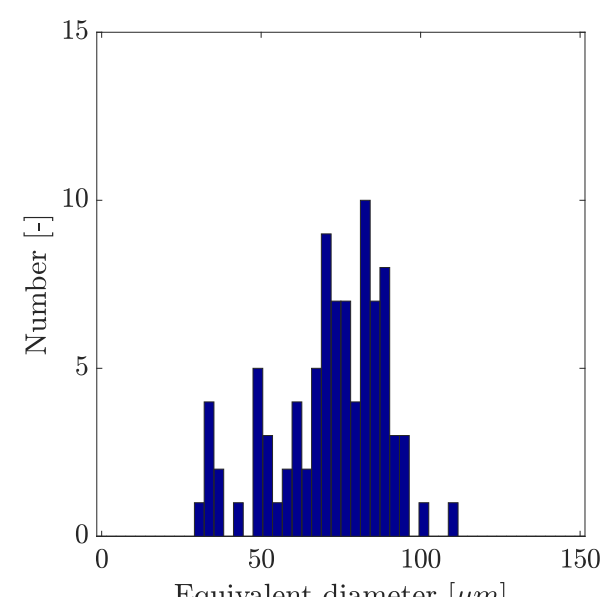

Figure 16: Distribution of the equivalent diameters of the glass beads

\subsection{Evolution of the diameters distribution with respect to the applied load}

Most of the porosities are quasi-spherical, and thus may be characterized by their equivalent diameter. More specifically, it is possible to compute the distribution of those equivalent diameters in the adhesive layer, and to track the evolution of this distribution along with the increase in the applied load. 


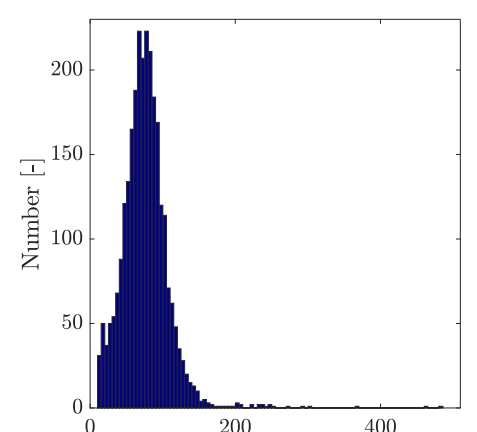

Equivalent diameter $[\mu \mathrm{m}]$

(a) $F=0 \mathrm{~N}$

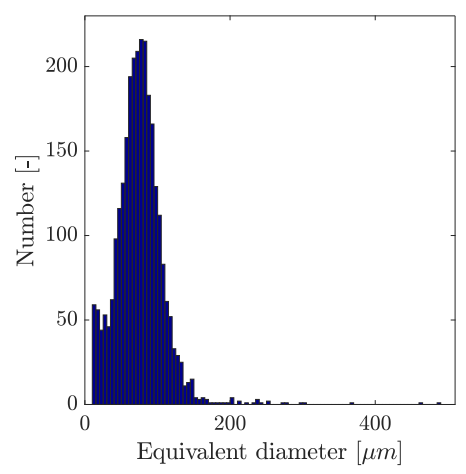

(c) $F=400 \mathrm{~N}$

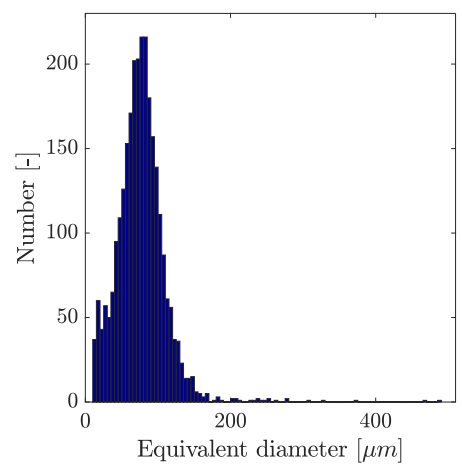

(e) $F=800 \mathrm{~N}$

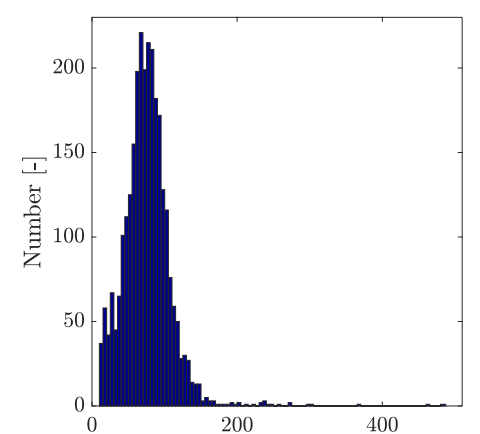

Equivalent diameter $[\mu m]$

(b) $F=200 \mathrm{~N}$

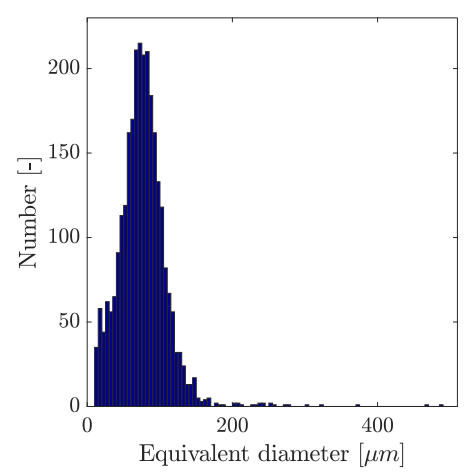

(d) $F=600 \mathrm{~N}$

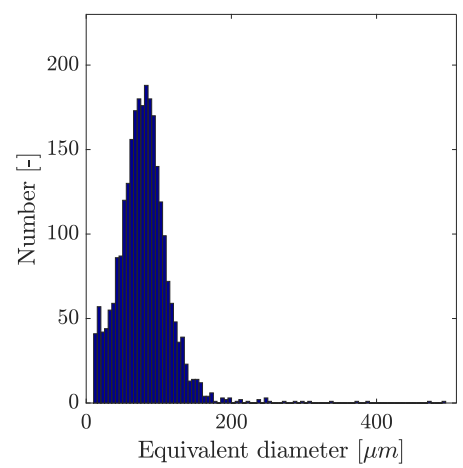

(f) $F=1000 \mathrm{~N}$

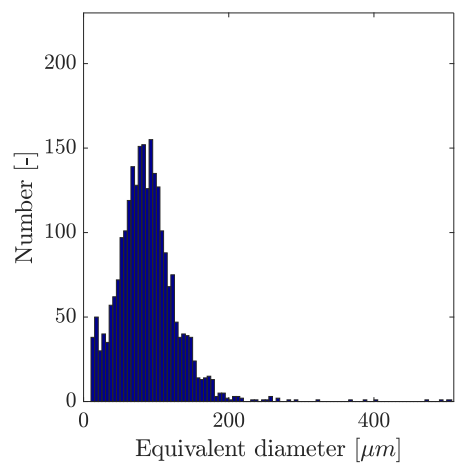

(g) $F=1100 \mathrm{~N}$

Figure 17: Effect on an increasing mechanical load on the equivalent diameters distribution 
It appears from Figs $17 \mathrm{a}$ to $17 \mathrm{~g}$ that the equivalent diameters distribution variations are somehow negligible for a load below 800 N. Starting from this load state (Figs $17 \mathrm{e}$ to 17g), the evolution becomes much clearer, as the distribution, which roughly follows a Gaussian shape, tends to widen.

To better visualize this trend, it is possible, for each distribution displayed in Fig.17, to fit a Gaussian distribution function (Eq, 6 to compute the mean equivalent diameter $\mu_{d}$ and the standard deviation $\sigma_{d}$.

$$
f(x)=\frac{1}{\sigma_{d} \sqrt{2 \pi}} \exp \left[-\left(\frac{x-\mu_{d}}{\sigma_{d}}\right)^{2}\right]
$$

This approach results in the data presented in Fig 18 .

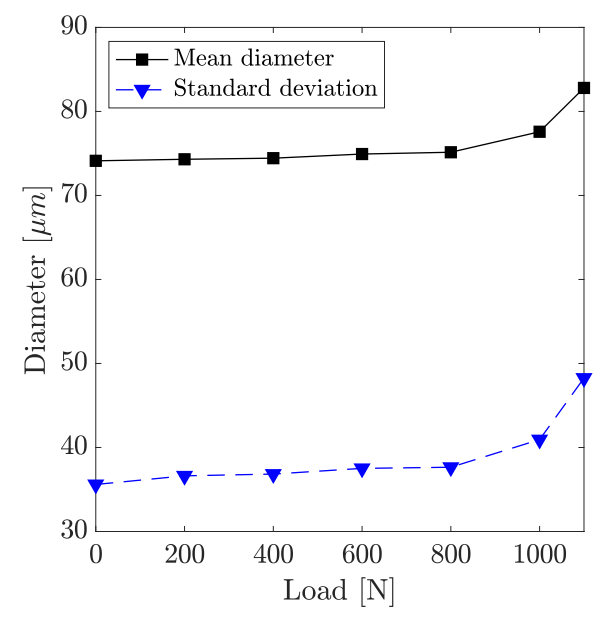

Figure 18: Parameters of the Gaussian distribution of the porosities diameters for every load investigated

A clearer trend can be seen in Fig 18 , which points out that even for the relatively low loads $(F<800$ $\mathrm{N}$ ), the microstructure of the adhesive undergoes slight, but visible nonetheless, transformations. Both the mean and the standard deviation of the distributions seem to follow the same trend. As it was expected from the data in Figs 15 and 17 from $F=800 \mathrm{~N}$ and higher, the distributions start to change significantly. The increase in size of the detected porosities may be explained by two factors: (i) the increasing load tends to open and expand the porosities (Fig $19 \mathrm{~d}$ ) and (ii) the increasing load may trigger coalescence phenomena, which cause neighboring porosities to merge (Figs 19a to 19c). These hypotheses are directly linked with the observations formulated regarding Figs 15 and 17, as they seem to be the most plausible explanation for these phenomena. 


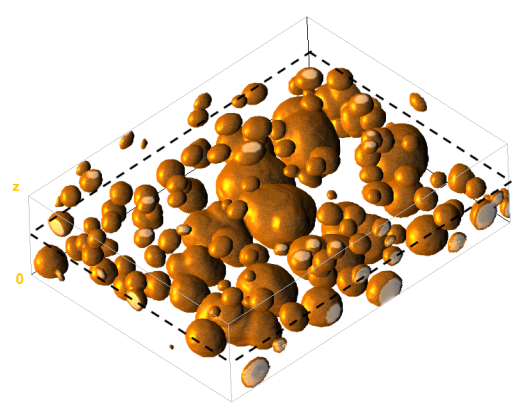

(a) Coalescence site, initial state (-- -: section plane)

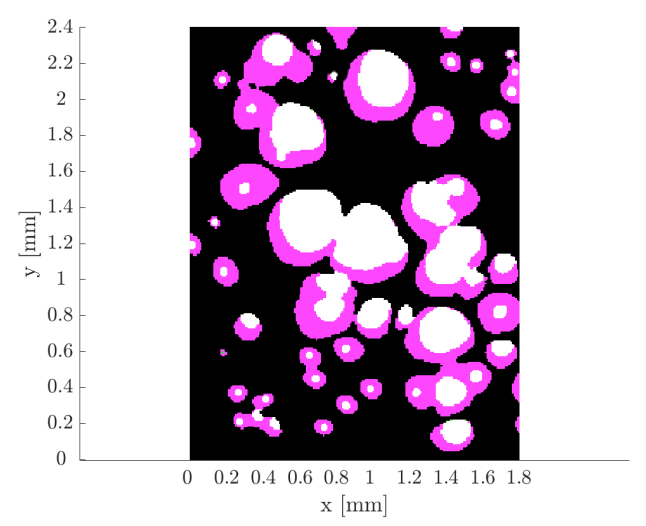

(c) Sliced view of coalescing porosities (white: initial state / magenta: final state)

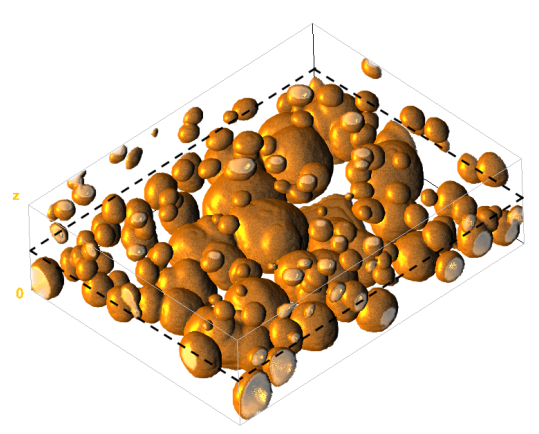

(b) Coalescence site, final state (-- -: section plane)

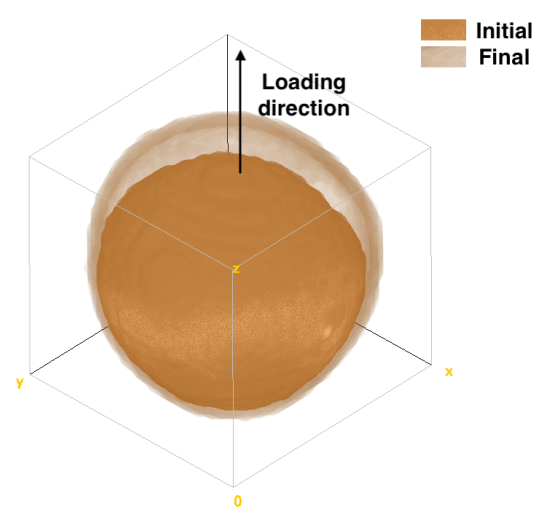

(d) Increase in volume $(F=0 \mathrm{~N}$ and $F=1100 \mathrm{~N})$ for an arbitrarily chosen porosity

Figure 19: Changes undergone by the porosities along with the increasing load

It is fairly easy to notice that some entities merged due to the application of the mechanical load, especially where the initial porosities were already close to each other. This is perfectly supported by the diminishing number of porosities detected by the processing tool, as it is shown in Fig $15 \mathrm{~b}$. Such merger phenomena were expected, as they have already been reported for other materials 21, 25].

The visualization of these simultaneous phenomena may be performed on the reconstructed volume using FiJi software [26], as presented in Fig.20. 


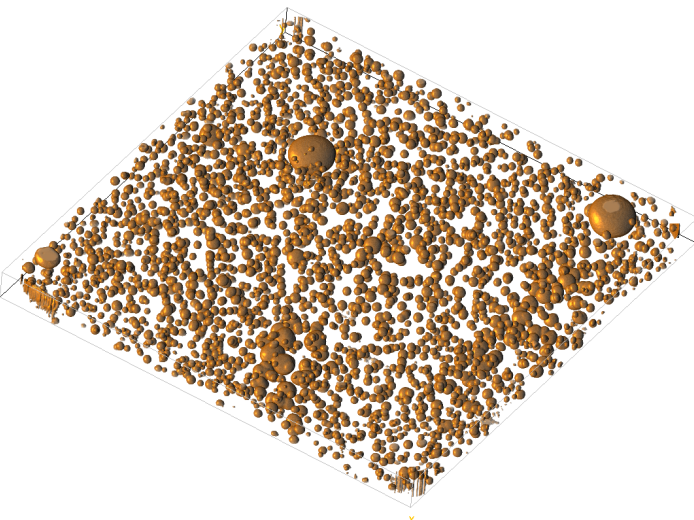

(a) Porosities field - Initial state

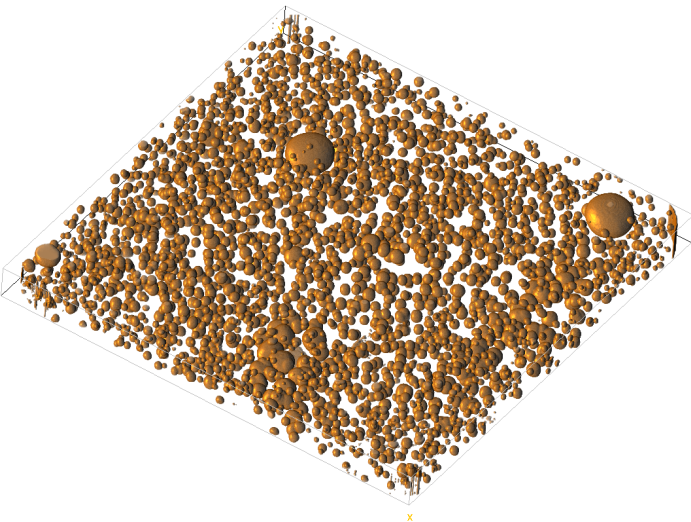

(b) Porosities field - Final state

Figure 20: 3D visualization of the porosities fields at initial and final states

The global increase in volume of the porosities in the adhesive is easy to visualize in Fig 20. This suggests that it is a phenomena which occur in the entierety of the joint, and not only in specific, predisposed locations. Moreover, it is possible to see in Fig 20 that there are a few locations where the porosities seem to be more concentrated. The adhesive bond is therefore weaker in these spots, and one may hypothesize that such concentrations of porosities could trigger mechanical failure mechanisms (microcracking, stress concentrations, localized plasticization, etc.) when stressed, and lead to the premature ruin of the assembly. Finally, the porosities in Fig 20 may be divided into two groups, depending on their size and incidence: (i) the most common porosities are rather small (a few tens of micrometers in terms of equivalent diameter) and they are created during the mixing of the adhesive, (ii) and the less usual porosities (in particular 2 specimens in Fig 20, easily identifiable), one order of magnitude bigger than the others (a few hundreds of micrometers in terms equivalent diameter), which are created during the spreading of the adhesive with the spatula on the adherends. This last type of porosities is the most critical threat to the mechanical strength of the joint, due to the size they feature. One might expect that if several of these large, but uncommon, porosities are located within a certain sphere of influence of each other (still to be determined), this could lead to an important and localized decrease in mechanical properties of the joint and once again be the cause of the unexpected mechanical ruin of the assembly.

\subsection{Sphericity of the porosities}

The porosities detected by the tomography measurements display for the most part a sphere-like shape, therefore a description based on the sphericity seems to be the best approach to characterize the evolution of their general shape. The sphericity $\Psi$ of an object is, as introduced by Wadell in 1935 [27, defined by the expression in Eq,7

$$
\Psi=\frac{\pi^{\frac{1}{3}}\left(6 V_{p}\right)^{\frac{2}{3}}}{A_{p}}
$$

where $V_{p}$ stands for the volume of the entity and $A_{p}$ for its area.

It is obvious from Eq, 7 that the sphericity varies theoretically between 0 (the studied entity being a plane) and 1 (the studied entity being a perfect sphere). 
The volume and the areas of the porosities being quantities easily accessible from the tomography data, the sphericity of each porosity may be computed, for various load states. The resulting data are plotted in Fig 21.

The technique employed in this paper is then able to detect the variation of the shape of each porosity, caused by the mechanical solicitation applied to the sample. It seems that the overall shape of the points clouds remains fairly the same, the most significant change in Fig 21 being the increase in equivalent diameters of the porosities, which tends to shift these points clouds to the right. Most of the computed sphericities are located around 1, which corroborates the observation according to which most of the porosities are quasi-spherical.

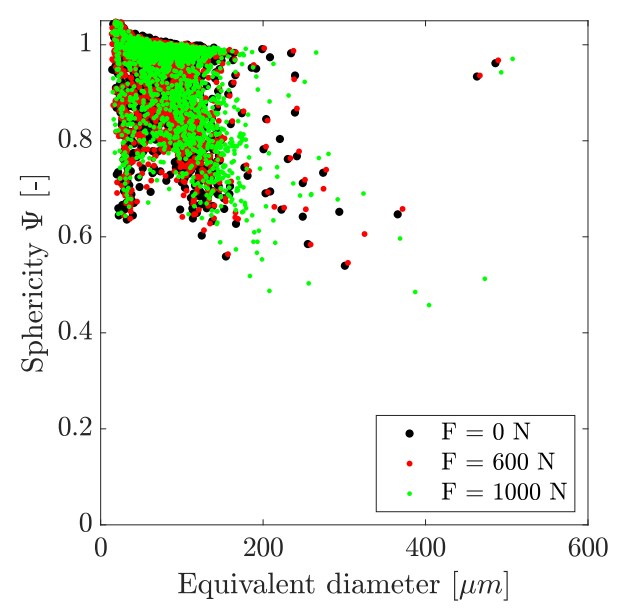

Figure 21: Sphericity of each detected porosity, for different load levels

One can see in Fig 21 that some points are located above the theoretical limit of 1, especially for the lowest equivalent diameters. This may be due to the effect of the voxelization during the measurements, as the voxel size is of $6 \mu \mathrm{m}$. For very small objects, this may induce seemingly incorrect sphericity values, due to areas miscalculations [28].

\section{Conclusion}

Porosities are commonplace defects in adhesive joints, formed during the mixing of the adhesive and during the bonding step. As such, adhesively bonded samples were made in order to investigate the porosity state within the bulk of their joints. The influence of tensile loadings on this porosity state was characterized using in-situ X-ray microtomography, until the mechanical failure of the samples.

In order to process the tomographic datasets, a processing tool was designed and calibrated on artificial datasets. This calibration was performed using optimization algorithms to tune the parameters of this processing tool: the size of kernel of the median filter, and the grayscale threshold used to identify the porosities in the reconstructed volumes. Two methods were proposed for this parameters optimization, and their performances were discussed. It was found that the best approach was to optimize the param- 
eters of the tool using both the porosities number and the porosity ratio.

Finally, it was experimentally proven that the application of a mechanical stress on the adhesive bonds has a significant influence on the porosities located in the medium. Once a certain stress is reached, coalescence phenomena are triggered, resulting in an abrupt decrease in porosities number. Moreover, due to the effect of the mechanical stress, the porosities tend to expand, resulting in an increasing porosity volumetric ratio. These changes in microstructure could be viewed as indicators that damage is occuring in the material. This is confirmed by the fact that the rate of growth significantly increase shortly before the fracture of the samples. Moreover, coalescence is occuring roughly for the same stress levels, leading to the possible relationship between these observed phenomena and mechanical damage.

These results could give new ways of improvements for models developed to predict the damage and the failure of the adhesively bonded joints. Indeed, it seems that the damage is due to a cavitation proecess (increase in the size of existing porosities or creation of new voids) and the failure is due to the coalescence of these porosities/voids. Thus, the effects of the triaxiality should be included in the damage model instead of only the out-of-plane stresses as commonly considered.

\section{References}

[1] R. D. Adams. Adhesive bonding science, technology and applications. Elsevier, 2005.

[2] L. F. M. da Silva. Modeling of Adhesively Bonded Joints. Springer-Verlag Berlin Heidelberg, 2005.

[3] Rolf Mahnken and Michael Schlimmer. "Simulation of strength difference in elasto-plasticity for adhesive materials". In: International Journal for Numerical Methods in Engineering 63.10 (2005), pp. $1461-1477$.

[4] J Y Cognard et al. "A study of the non-linear behaviour of adhesively-bonded composite assemblies". In: (2006).

[5] J Y Cognard et al. "Analysis of the nonlinear behavior of adhesives in bonded assemblies-Comparison of TAST and Arcan tests". In: International Journal of Adhesion 8 Adhesives 28 (2008), pp. 393404.

[6] Julien Maurice et al. "Characterization and modelling of the 3D elastic-plastic behaviour of an adhesively bonded joint under monotonic tension/compression-shear loads: Influence of three cure cycles". In: Journal of Adhesion Science and Technology - J ADHES SCI TECHNOL 27 (Jan. 2012), pp. 1-17.

[7] J.Y. Cognard, R. Créac'hcadec, and J. Maurice. "Numerical analysis of the stress distribution in single-lap shear tests under elastic assumptionApplication to the optimisation of the mechanical behaviour". In: International Journal of Adhesion and Adhesives 31.7 (2011), pp. 715-724.

[8] Nicolas Carrere et al. "3D models of specimens with a scarf joint to test the adhesive and cohesive multi-axial behavior of adhesives". In: International Journal of Adhesion and Adhesives 62 (2015), pp. $154-164$. 
[9] C. Badulescu et al. "Analysis of the low temperature-dependent behaviour of a ductile adhesive under monotonic tensile/compressionshear loads". In: International Journal of Adhesion and Adhesives 36 (2012), pp. 56-64.

[10] J. Y. Buffiere et al. "In Situ Experiments with X ray Tomography: An Attractive Tool for Experimental Mechanics". In: Experimental Mechanics 50 (Mar. 2010), pp. 289-305.

[11] H. Toda et al. "In situ observation of ductile fracture using X-ray tomography technique". In: Acta Materialia 59.5 (2011), pp. 1995-2008.

[12] A. Bouterf et al. "Identification of the crushing behavior of brittle foam: From indentation to oedometric tests". In: Journal of the Mechanics and Physics of Solids (2017).

[13] Stéphane Roux et al. "Three-dimensional image correlation from X-ray computed tomography of solid foam". In: Composites Part A: Applied Science and Manufacturing (2008).

[14] T.F. Morgeneyer, M.J. Starink, and I. Sinclair. "Evolution of voids during ductile crack propagation in an aluminium alloy sheet toughness test studied by synchrotron radiation computed tomography". In: Acta Materialia 56.8 (2008), pp. 1671-1679.

[15] Alireza Bastani et al. "Study of adhesive bondlines in modified wood with fluorescence microscopy and X-ray micro-computed tomography". In: International Journal of Adhesion and Adhesives 68 (2016), pp. 351-358.

[16] Philip D. Evans et al. "Visualization and numerical analysis of adhesive distribution in particleboard using X-ray micro-computed tomography". In: International Journal of Adhesion and Adhesives 30.8 (2010), pp. $754-762$.

[17] Jesse L. Paris and Frederick A. Kamke. "Quantitative woodadhesive penetration with X-ray computed tomography". In: International Journal of Adhesion and Adhesives 61 (2015), pp. 71-80.

[18] B. Pollak. "Experiences with Planography". In: Diseases of the Chest 24.6 (1953), pp. 663 -669.

[19] Avinash C. Kak and Malcolm Slaney. Principles of computerized tomographic imaging. Philadelphia: Society for Industrial and Applied Mathematics, 2001.

[20] L A Feldkamp, L C Davis, and J W Kress. Practical cone-beam algorithm. Tech. rep. 6. 1984.

[21] B. R. Pinzer et al. "3D-characterization of three-phase systems using X-ray tomography: tracking the microstructural evolution in ice cream". In: Soft Matter 8.17 (2012), p. 4584.

[22] A. Ilioni et al. "A viscoelastic-viscoplastic model to describe creep and strain rate effects on the mechanical behaviour of adhesively-bonded assemblies". In: International Journal of Adhesion and Adhesives 82 (2018), pp. 184-195.

[23] J. S. Lim. "Two-dimensional signal and image processing". In: (1990), pp. 469-476.

[24] Neal C. Gallagher and Gary L. Wise. "A Theoretical Analysis of the Properties of Median Filters". In: IEEE Transactions on Acoustics, Speech, and Signal Processing 29.6 (1981), pp. 1136-1141.

[25] H. Klocker and V. Tvergaard. "Void growth and coalescence in metals deformed at elevated temperature". In: International Journal of Fracture 106.3 (2000), 259276. 
[26] Johannes Schindelin et al. "Fiji: an open-source platform for biological-image analysis". In: Nature Methods 9 (June 2012), $676 \mathrm{EP}-$.

[27] Hakon Wadell. "Volume, Shape, and Roundness of Quartz Particles". In: The Journal of Geology 43.3 (1935), pp. 250-280.

[28] Gaëtan Lehmann, David Legland, and U M R Génie. "Efficient N-Dimensional surface estimation using Crofton formula and run-length encoding". In: The Insight Journal 2 (2012), pp. 1-11. 